\title{
The Anti-dementia Effects of Donepezil Involve miR-206-3p in the Hippocampus and Cortex
}

\author{
Cheng-Niu Wang, ${ }^{a, \#}$ Ying-Jie Wang, ${ }^{b, c, \#}$ Hao Wang, ${ }^{b, c}$ Lu Song,,${ }^{b, c}$ Yu Chen, ${ }^{d}$ Jin-Liang Wang, ${ }^{b, c}$ \\ Ying Ye, ${ }^{b, c}$ and Bo Jiang* ${ }^{*, b}$ \\ ${ }^{a}$ Basic Medical Research Centre, Medical College, Nantong University; Nantong 226001, Jiangsu, China: \\ ${ }^{b}$ Department of Pharmacology, Pharmacy College, Nantong University; Nantong 226001, Jiangsu, China: ${ }^{c}$ Provincial \\ Key Laboratory of Inflammation and Molecular Drug Target; Nantong 226001, Jiangsu, China: and ${ }^{d}$ Shanghan \\ Teaching and Research Section, Clinical College of Chinese Medicine, Hubei University of Traditional Chinese \\ Medicine; Wuhan 430065, China.
}

Received November 18, 2016; accepted January 10, 2017; advance publication released online January 25, 2017

\begin{abstract}
Alzheimer's disease (AD) is a most serious age-related neurodegenerative disorder accompanied with significant memory impairments in this world. Recently, microRNAs (miRNAs) have been reported to be invlolved in the pathophysiology of AD. Previous studies have shown that miRNA-206 (miR-206) is implicated in the pathogenesis of AD via suppressing the expression of brain-derived neurotrophic factor (BDNF) in the brain. Here, we examined the miR-206-3p and miR-206-5p expression in the hippocampus and cortex of Abeta precursor protein (APP)/presenilin-1 (PS1) transgenic mice treated with donepezil, a drug approved for treating AD in clinic. We found that the expression of miR-206-3p was significantly up-regulated in the hippocampus and cortex of APP/PS1 mice, while donepezil administration significantly reversed this dysfunction. In addition, enhancing the miR-206-3p level by the usage of AgomiR-206-3p significantly attenuated the anti-dementia effects of donepezil in APP/PS1 mice. Together, these results suggested that miR-206-3p is involved in the anti-dementia effects of donepezil, and could be a novel pharmacological target for treating AD.
\end{abstract}

Key words Alzheimer's disease; brain-derived neurotrophic factor; donepezil; microRNA (miRNA)-206-3p

Alzheimer's disease (AD) is a serious neurodegenerative disorder in old people, and always accompanied with significant memory impairments, affecting mainly the hippocampus and frontal cortex. ${ }^{1,2)} \mathrm{AD}$ patients display relatively slow, chronic but progressive neurodegeneration and memory impairments, ultimately leading to full dementia. ${ }^{3,4)}$ Recent survey found that AD is responsible for about $50-60 \%$ of all cases of dementia in people over age $65^{3,4)}$

The etiology of AD remains unclear, though many pathophysiologic hallmarks of this disease have been disclosed and are currently well established. They involve a complex network of interconnected factors such as cholinergic dysfunction, aggregation and accumulation of $\beta$-amyloid $(\mathrm{A} \beta)$, intracellular formation of neurofibrillary tangles composed by tau, oxidative stress, neuronal loss, and so on. ${ }^{5-8)} \mathrm{A}$ lot of reports have shown that brain-derived neurotrophic factor (BDNF) is critical for the establishment and maintenance of memory, while the dysfunction of BDNF system leads to memory impairments. ${ }^{9,10)}$ It is known that BDNF combines to tyrosine kinase receptor $\mathrm{B}$ (TrkB) in the cell membrane, and then inducing the activation of several intracellular signaling pathways. ${ }^{1-13)}$ Accumulating evidences have indicated that BDNF is implicated in the pathophysiology of AD. Previous studies have already found the reduced expression of BDNF in several brain regions (especially the hippocampus) of postmortem AD samples. ${ }^{14,15)}$ By using the immunohistochemical method, Connor et al. found a significant reduction in not only intensity, but also the number of BDNF-immunoreactive cell in the hippocampus and cortex of AD samples. ${ }^{16)}$ In addition, it was

\# These authors contributed equally to this work. also thought that the $\mathrm{A} \beta$-induced neurotoxicity and dendrite atrophy may due to a consequence of BDNF deficiency. ${ }^{17,18)}$

Donepezil is one of the only four drugs currently approved for treatment of Alzheimer's dementia. Although thought as a selective acetylcholinesterase inhibitor, the precise antidementia mechanism of donepezil in patients with $\mathrm{AD}$ is not fully understood. ${ }^{19,20)}$ Leyhe et al. and Alvarez et al. have shown that donepezil increase the serum concentration of BDNF in AD patients. ${ }^{21,22)}$ Autio et al. also revealed that donepezil administration led to rapid phosphorylation of TrkB in the hippocampus. ${ }^{23)}$

MicroRNAs (miRNAs) are single-stranded small noncoding RNA molecules which interact with the 3 '-untranslated region (UTR) of specific target mRNAs to modulate gene expression. ${ }^{24)}$ MiRNAs regulate a lot of biological processes, and are also involved in many diseases. MiRNA-206 (miR-206) is initially known to be abundant in muscle tissue and closely involved in muscle development. ${ }^{25)}$ Recently, more and more studies have been demonstrating the role of miR-206 in the brain. Previous reports have already shown that AD patients and AD models all have high level of miR-206 in the brain, which contributes to memory impairments by suppressing the BDNF expression in the brain. ${ }^{26-28)}$ Thus it is possible that donepezil produces anti-dementia actions by regulating the level of miR-206 in the brain. Here, we studied this speculation using APP/PS1 transgenic mice, behavioral test, and quantitative real-time reverse transcription PCR method.

\section{MATERIALS AND METHODS}

Animals The Abeta precursor protein (APP)/presenilin-1

* To whom correspondence should be addressed. e-mail: jiangbo78099@ntu.edu.cn 

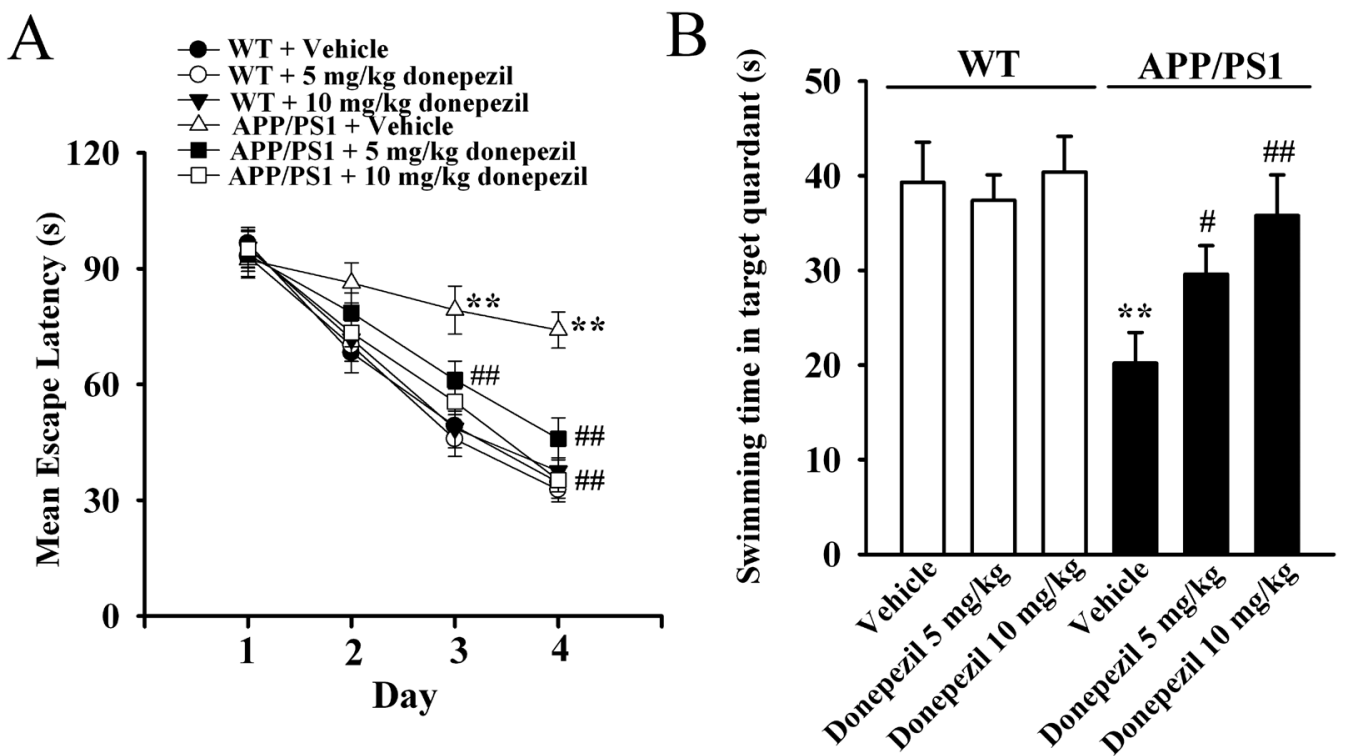

Fig. 1. In the Morris Water Maze, Donepezil Treatment Restores the Memory Impairments in APP/PS1 Mice

(A) Comparison of the mean escape latency during the training trials for vehicle-treated WT mice, $5 \mathrm{mg} / \mathrm{kg}$ donepezil-treated WT mice, $10 \mathrm{mg} / \mathrm{kg}$ donepezil-treated WT mice, vehicle-treated APP/PS1 mice, $5 \mathrm{mg} / \mathrm{kg}$ donepezil-treated APP/PS1 mice, and $10 \mathrm{mg} / \mathrm{kg}$ donepezil-treated APP/PS1 mice. (B) Comparison of the swimming time spent in the target quadrant during the probe test for each group. Data are expressed as means \pm S.E.M. $(n=10) ; * * p<0.01 v s$. WT + Vehicle; ${ }^{\#} p<0.05,{ }^{\#} p<0.01 v s$. APP/PS1+Vehicle.
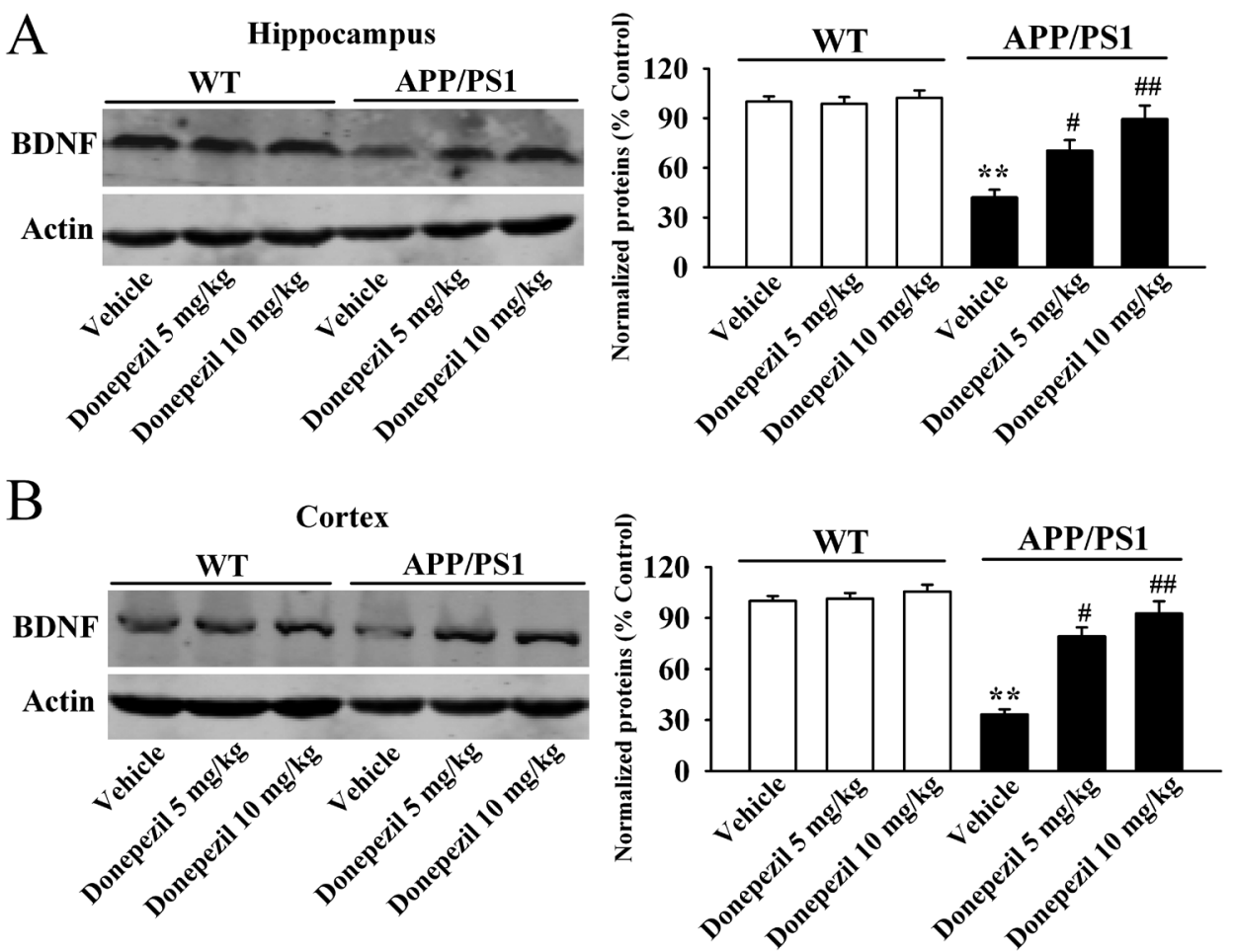

Fig. 2. Donepezil Treatment Restores the Decreased Hippocampal and Cortical BDNF Protein Expression in APP/PS1 Mice

(A) APP/PS1 mice displayed significantly less BDNF protein expression in the hippocampus than WT control mice, while this dysfunction was fully reversed by donepezil administration. (B) Similar to hippocampus, donepezil treatment also restored the decreased BDNF protein expression in the cortex of APP/PS1 mice. Data are expressed as means \pm S.E.M. $(n=5) ;{ }^{* *} p<0.01$ vs. WT+Vehicle; ${ }^{\#} p<0.05,{ }^{\# \#} p<0.01 v$ s. APP/PS1+Vehicle.

(PS1) transgenic mice used in this study were male, tenmonth-old, and developed in a C57BL/6J background. All the APP/PS1 mice and littermate wild-type (WT) mice were provided by the Model Animal Research Center of Nanjing University (China). Before experiments started, all the mice were housed 5 per cage for one week under standard conditions (free access to food and water; lights from 07:00 to 19:00; room temperature $24 \pm 1^{\circ} \mathrm{C}$ ), as we previously described. ${ }^{29-32}$ ) All the behavioral experiments were performed during the day. All the procedures involving mice were conducted in accordance with the National Institutes of Health Guide for Care and Use of Laboratory Animals and with the Euro- 
pean Communities Council Directive of 24 November 1986 (86/609/EEC).

Drugs and Treatments Donepezil was provided by Tocris (Bristol, U.K.) and dissolved in 0.9\% saline. AgomiR-206-3p was provided by RiboBio (Guangzhou, China). The dosage of donepezil $(5,10 \mathrm{mg} / \mathrm{kg})$ and AgomiR-206-3p (100 nmol/ $\mathrm{kg})$ were chosen based on previous reports. ${ }^{29,33,34)}$ Donepezil was administered intraperitoneally (i.p.) in a volume of $10 \mathrm{~mL} / \mathrm{kg}$, while AgomiR-206-3p was tail-vein injected.

Morris Water Maze The Morris water maze was conducted as we previously did. ${ }^{29,35)}$

Western Blotting Analysis The Western blotting method was also performed as we previously did. $^{29-32)}$

Quantitative Real-Time Reverse Transcription PCR (qRT-PCR) Dectection The miR-206-3p, miR-206-5p, and BDNF mRNA levels were determined using qRT-PCR method. ${ }^{28,36-38)}$ For the detection of miR-206, total miRNAs were isolated from the hippocampus and cortex using the mirVana miRNA isolation kit (Ambion, CA, U.S.A.). After the measurement of RNA quantity, the mirVana qRT-PCR miRNA kit (Ambion) was further used per the kit instructions. U6 was selected as the internal control. For the detection of BDNF mRNA, total RNA was isolated from the hippocampus and cortex using the TRIzol ${ }^{\circledR}$ reagent (Thermo Fisher, DE, U.S.A.), and the RT system (Promega, Madison, WI, U.S.A.) and SYBR $^{\circledR}$ fast qPCR master mix (TaKaRa, Japan) were further used following the kit protocols. Glyceraldehyde-3-phosphate dehydrogenase (GAPDH) was selected as the internal control. Each sample was assayed in triplicate. The primers for miR-206-3p (RT primer: 5'-GTC TGT ATG GTT GTT CTG CTC TCT GTC TCA TCC CTA TCT ACAACCATACAGACCCACACACAT-3'; PCR forward primer: 5'-CTGCCGTGGAAT GTA AGGAA-3'; PCR reverse primer: 5'-TAT GGT TGT TCT GCTCTC TGTCTC-3'), miR-206-5p (RT primer: 5'-GTC TGT ATG GTT GTT CAC GAC TCC TTC ACA TCC CTA TCC AAC CAT ACA GAC TAT GAGGAT A-3'; PCR forward primer: 5'-GCTGCTCAGACATGCTTCTTTATA-3'; PCR reverse primer: 5'-TATGGTTGT TCACGACTCCTT CAC-3'), and U6 (RT primer: 5'-GTCGTA TCC AGT GCA GGG TCG AGG TGC ACT GGA TAC GAC AAA ATA TGG-3'; PCR forward primer: 5'-ATT GGA ACGATA CAG AGA AGA TT-3'; PCR reverse primer: 5'-GGA ACGCTTCACGAA TTT G-3') were designed by GenePharma (Shanghai, China). The primers for BDNF mRNA (PCR forward primer: 5'-GACAAG GCAACT TGGCCT AC-3'; PCR reverse primer: 5'-CCT GTC ACA CAC GCT CAGCTC-3') and GAPDH (PCR forward primer: 5'-ACA TTG TTGCCA TCA ACG AC-3'; PCR reverse primer: 5'-ACGCCA GTA GAC TCCACGAC-3') were designed by Sangon Biotech (Shanghai, China). The threshold cycle $\left(\mathrm{C}_{\mathrm{T}}\right)$ was defined as the fractional cycle number at which the fluorescence passed the fixed threshold. The relative gene expression levels of miR-206-3p, miR-206-5p, and BDNF mRNA were normalized to their internal controls, and calculated using the $\triangle \triangle \mathrm{C}_{\mathrm{T}}$ method.

Statistical Analysis All the data analysis was performed using two-way ANOVA followed by post-hoc Bonferroni's test (SPSS 13.0 software). $p<0.05$ level was thought statistically significant in this study.
A

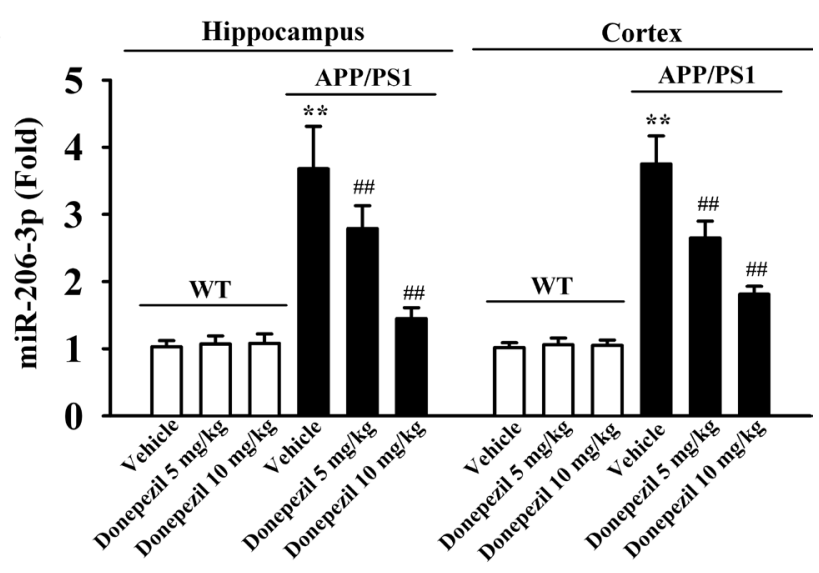

B

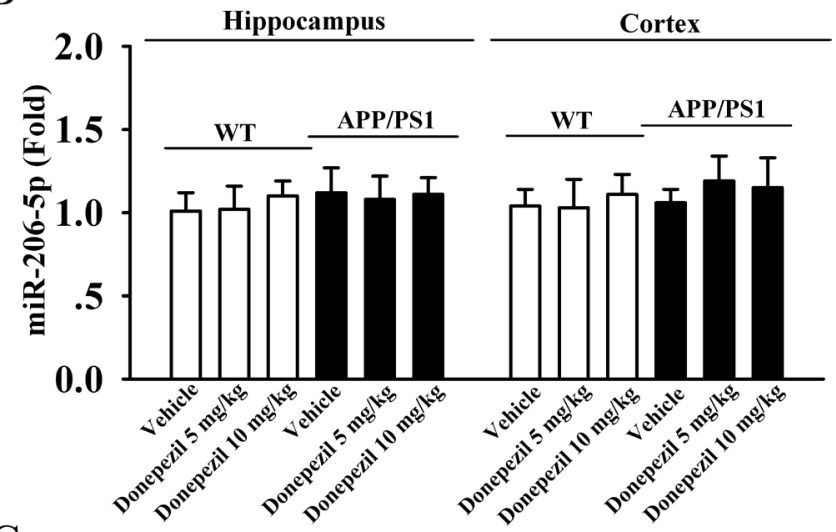

C

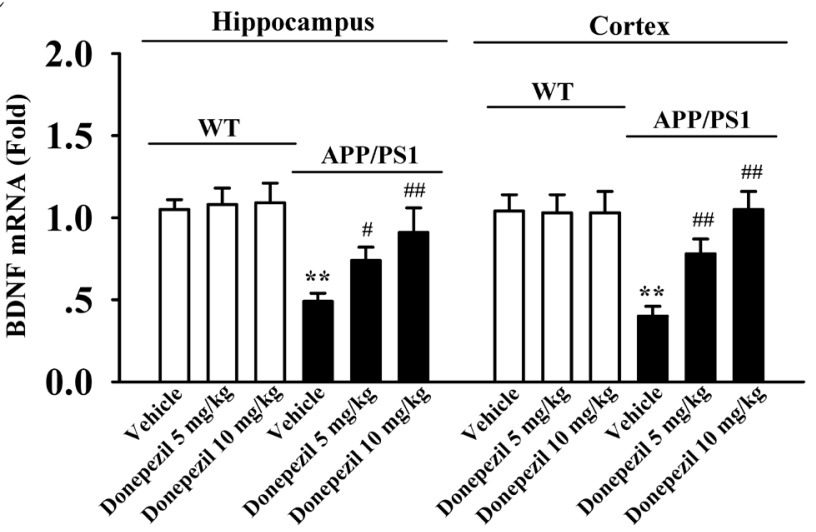

Fig. 3. Donepezil Administration Reverses the Dysfunction in miR206-3p and BDNF mRNA Levels in APP/PS1 Mice

(A) APP/PS1 mice displayed significantly higher miR-206-3p level in the hippocampus and cortex than WT control mice, while donepezil treatment fully restored this dysfunction. (B) There were no significant changes in the hippocampal and cortical miR-206-5p level between all the groups. (C) In contrast to miR-206-3p APP/PS1 mice had significantly less BDNF mRNA level in the hippocampus and cortex than WT control mice, while donepezil treatment also reversed this dysfunction. Data are expressed as means \pm S.E.M. $(n=5) ; * * p<0.01 v s$. WT+Vehicle; ${ }^{\#} p<0.05,{ }^{\#} p<0.01$ vs. APP/PS1+Vehicle.

\section{RESULTS}

Chronic Donepezil Administration Restores the Memory Impairments and BDNF Dysfunction in APP/PS1 Mice As a first step of this study, both the APP/PS1 and WT mice were administrated with donepezil or vehicle for $10 \mathrm{~d}$, with the Morris water maze performed during the last five days. Mice were injected with donepezil/vehicle $30 \mathrm{~min}$ before the first learning trial of each training day. The behavioral data was 

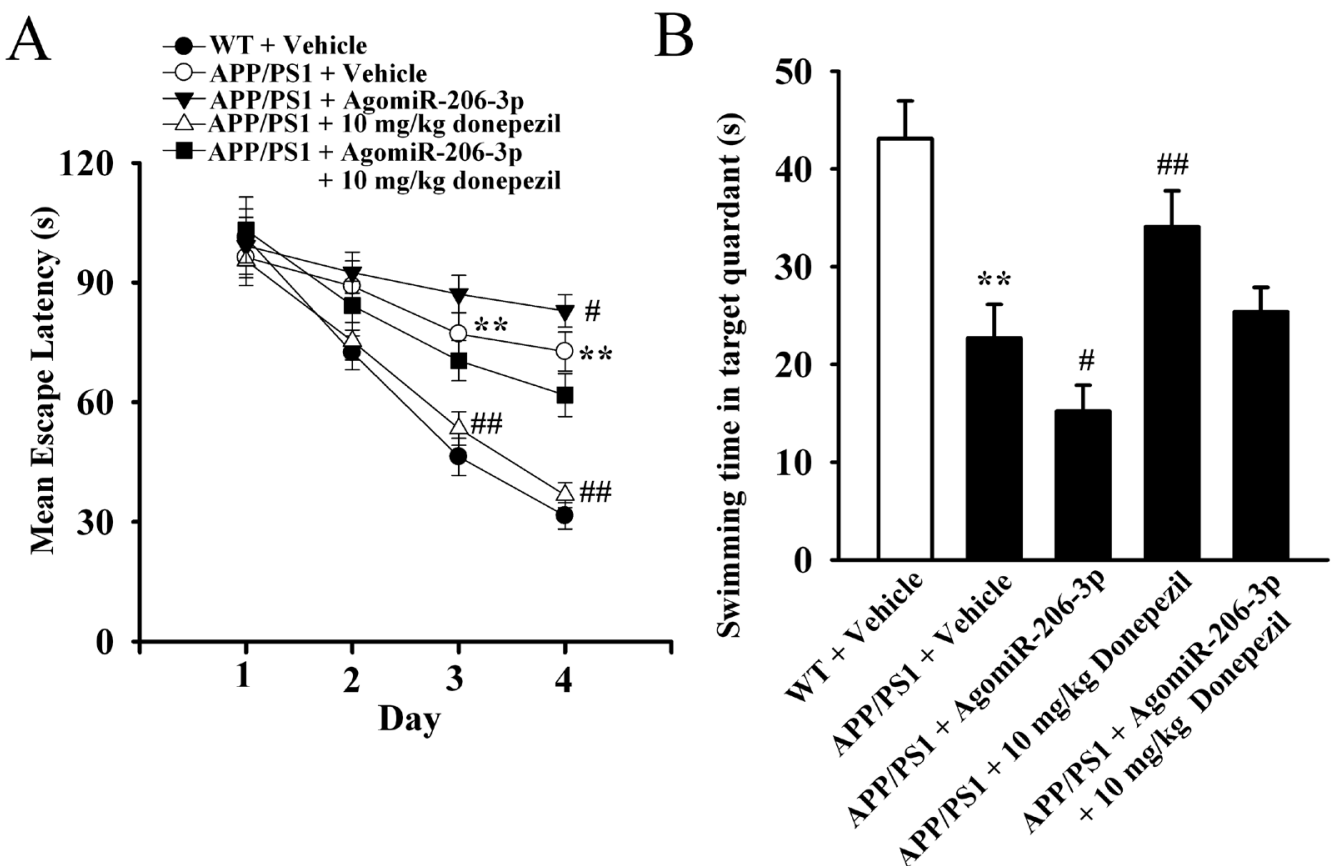

Fig. 4. The Usage of AgomiR-206-3p Attenuates the Anti-dementia Effects of Donepezil in the Morris Water Maze

(A) Comparison of the mean escape latency during the training trials for vehicle-treated WT mice, vehicle-treated APP/PS1 mice, AgomiR-206-3p-treated APP/PS1 mice, $10 \mathrm{mg} / \mathrm{kg}$ donepezil-treated APP/PS1 mice, and AgomiR-206-3p+10 mg $/ \mathrm{kg}$ donepezil co-treated APP/PS1 mice. (B) Comparison of the swimming time spent in the target quadrant during the probe test for each group. Data are expressed as means \pm S.E.M. $(n=10) ; *^{*} p<0.01 v s$. WT + Vehicle; ${ }^{\#} p<0.05$, ${ }^{\#} p<0.01 v s$. APP/PS1+Vehicle.

summarized in Fig. 1. It was found that the vehicle-treated APP/PS1 mice displayed significantly longer escape latency than vehicle-treated WT mice in the 3rd and 4th training day (Fig. 1A, $n=10, p<0.01 v s$. WT+Vehicle), proving the effectiveness and reliability of our mice model of AD. It was also found that $5 \mathrm{mg} / \mathrm{kg}$ donepezil treatment reduced the escape latency of APP/PS1 mice starting from the 2nd training day, while $10 \mathrm{mg} / \mathrm{kg}$ donepezil-treated APP/PS1 mice displayed almost the same level of escape latency to vehicle-treated WT mice during all the 4 training days (Fig. 1A, $n=10, p<0.01 v s$. APP/PS1+Vehicle). Similarly, APP/PS1 mice exhibited significantly memory impairments in the probe test (Fig. $1 \mathrm{~B}, n=10$, $p<0.01 v s$. WT+Vehicle), while $10 \mathrm{mg} / \mathrm{kg}$ donepezil administration fully reversed this dysfunction (Fig. 1B, $n=10, p<0.01$ vs. APP/PS1+Vehicle). Moreover, donepezil administration did not affect the memory ability of WT control mice in the Morris water maze $(n=10)$.

Next, Western blotting experiments were performed to detect the BDNF expression in the hippocampus and cortex, with $\beta$-actin used as the internal control. As shown in Fig. 2, the hippocampal and cortical BDNF expression in APP/PS1 mice were significantly less than that in WT control mice ( $n=5, p<0.01$ vs. WT+Vehicle), consistent with previous findings. ${ }^{28,39,40)}$ In line with the behavioral data, chronic donepezil administration significantly enhanced the expression of hippocampal and cortical BDNF in APP/PS1 mice $(n=5, p<0.01$ $v s$. APP/PS1+Vehicle). Donepezil treatment did not affect the BDNF expression in WT control mice $(n=5)$.

Chronic Donepezil Treatment Reverses the Enhanced miR-206-3p Level in APP/PS1 Transgenic Mice Furthermore, qRT-PCR experiments were done to examine the levels of miR-206-3p and miR-206-5p in the hippocampus and cortex of vehicle-treated WT mice, donepezil-treated
WT mice, vehicle-treated APP/PS1 mice, and donepeziltreated APP/PS1 mice. As shown in Fig. 3A, APP/PS1 mice displayed significantly higher miR-206-3p level in both the hippocampus and cortex than WT control mice $(n=5, p<0.01$ vs. WT+Vehicle), consistent with previous finding. ${ }^{27,28)}$ Detailed analysis revealed that the hippocampal miR-206-3p level in APP/PS1+vehicle mice was about $257.3+17.3 \%$ higher than that in WT control+vehicle mice, while the cortical miR-206-3p level in APP/PS1 mice was about 267.6+21.4\% higher than that in WT control+vehicle mice. Interestingly, the usage of donepezil fully down-regulated the miR-206-3p levels in APP/PS1 mice ( $n=5, p<0.01 v s$. APP/PS1+Vehicle). Detailed analysis also indicated that the hippocampal miR-206-3p level in APP/PS1 $+10 \mathrm{mg} / \mathrm{kg}$ donepezil mice was about $60.6+11.2 \%$ less than that in APP/PS1+vehicle mice, while the cortical miR-206-3p level in APP/PS1 $+10 \mathrm{mg} / \mathrm{kg}$ donepezil mice was about $51.7+10.4 \%$ less than that in APP/PS1+vehicle mice. Figure 3B illustrated the miR-206-5p data, and however, it was found that there was no significant change in the miR-206-5p level among all the groups $(n=5)$. In addition, donepezil treatment did not influence the miR-206-3p and miR-206-5p levels in WT control mice $(n=5)$. These data suggest that donepezil may produce anti-dementia effects through down-regulating the hippocampal and cortical miR-206-3p.

Correspondingly, the BDNF mRNA level in each group was also examined, and the data was shown in Fig. 3C. The change for BDNF mRNA in each group was in line with that for BDNF protein, while in contrast to that for miR-206-3p. It was found that APP/PS1 mice had significantly less BDNF mRNA in the hippocampus and cortex than WT control mice $(n=5, p<0.01 \quad v s$. WT+Vehicle), while chronic donepezil treatment fully reversed this dysfunction $(n=5, p<0.01$ vs. APP/PS1+Vehicle). 
A

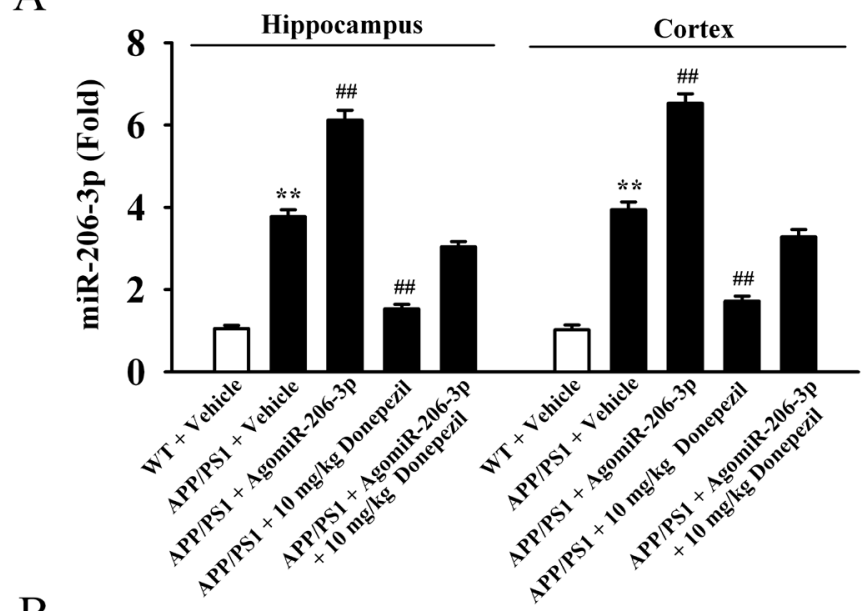

B

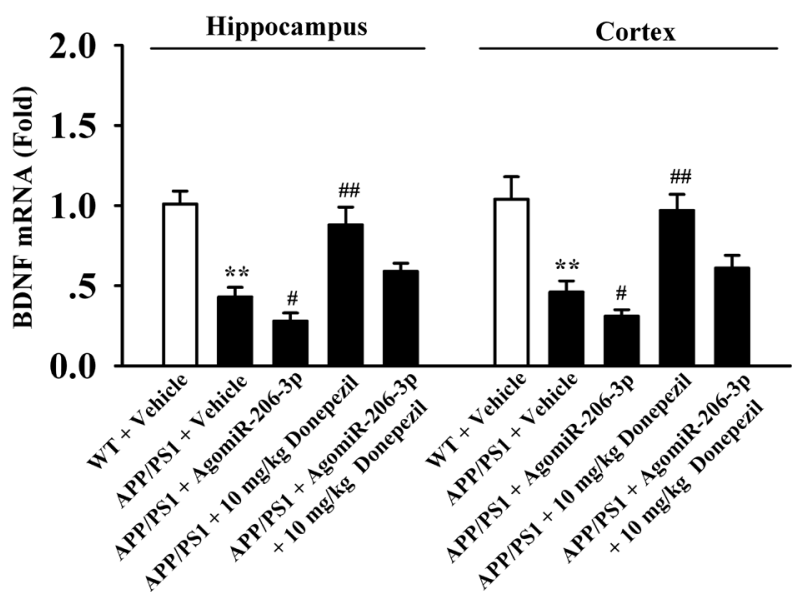

Fig. 5. The Usage of AgomiR-206-3p Attenuates the Effects of Donepezil on miR-206-3p and BDNF mRNA

(A) qRT-PCR results showed that AgomiR-206-3p pretreatment significantly prevented the donepezil-induced decrease of hippocampal and cortical miR-206-3p in APP/PS1 mice. (B) qRT-PCR results showed that AgomiR-206-3p pretreatment also significantly prevented the donepezil-induced increase of hippocampal and cortical BDNF mRNA in APP/PS1 mice. Data are expressed as means \pm S.E.M. $(n=5)$ ${ }_{* *}^{*} p<0.01$ vs. WT+Vehicle; ${ }^{\#} p<0.05,{ }^{\#} p<0.01 v s$. APP/PS1+Vehicle.

The Anti-dementia Effects of Donepezil in APP/PS1 Mice Were Attenuated by miR-206-3p Enhancing To further explore the relationship between miR-206-3p and the antidementia effects of donepezil, AgomiR-206-3p was used to enhance the level of miR-206-3p in the brain. APP/PS1 mice were co-administrated with AgomiR-206-3p (30 min before donepezil treatment) and donepezil for $10 \mathrm{~d}$, with the Morris water maze performed during the last five days. The behavioral data are summarized in Figs. 4A and B, and we can see that compared to vehicle-treated APP/PS1 mice, the usage of AgomiR-206-3p further exacerbated the memory impairments in APP/PS1 mice ( $n=10, p<0.01 v s$. APP/PS1+Vehicle). Importantly, AgomiR-206-3p pretreatment significantly attenuated the anti-dementia effects of donepezil in the tests $(n=10)$.

After the behavioral tests, qRT-PCR and Western blotting experiments were also performed. The qRT-PCR results showed that AgomiR-206-3p+donepezil co-treated APP/PS1 mice displayed significantly higher miR-206-3p level and lower BDNF mRNA level in the hippocampus and cortex than donepezil-treated APP/PS1 mice (Figs. 5A, B, $n=5$ ). The Western blotting results revealed that AgomiR-206-3p+donepezil co-treated APP/PS1 mice displayed significantly lower BDNF protein expression in the hippocampus and cortex than donepezil-treated APP/PS1 mice (Figs. 6A, B, $n=5$ ). Collectively, these results suggest that the anti-dementia effects of donepezil were mediated through down-regulating the miR-206-3p level in the brain.

\section{DISCUSSION}

Here, we reported that chronic donepezil administration not only improved the memory abilities and BDNF dysfunction in APP/PS1 mice, but also down-regulated the miR-206-3p expression in the hippocampus and cortex.

The anti-dementia effects of donepezil in AD animal models have already been demonstrated. In this study, we used the classic behavior test, Morris water maze, to assess the effects of donepezil in APP/PS1 mice model of AD. Consistent with previous reports, ${ }^{41-43)}$ donepezil produced significant beneficial effects on the memory ability of APP/PS1 mice, suggesting the effectiveness of $\mathrm{AD}$ model and donepezil used in this study.

Although thought as a centrally acting reversible acetylcholinesterase inhibitor, more and more pharmacological effects of donepezil are being explored. For example, Ye et al. reported that donepezil reduced the $\mathrm{A} \beta$ accumulation in mitochondria. ${ }^{44)}$ Guo et al. found that donepezil improved the cognitive deficits in APP/PS1 mice by inhibiting microglial activation and the release of pro-inflammatory cytokines. ${ }^{42)}$ Solntseva et al. also showed that activation of sigma-1 receptor was involved in the protective effects of donepezil against the $\mathrm{A} \beta$-induced hippocampal long-term potentiation (LTP) impairments. ${ }^{45)}$ In our study, the finding that donepezil administration reversed the BDNF dysfunction in APP/PS1 mice may extend the clinical application of donepezil, as BDNF is also involved in some other neurological disorders.

How does donepezil reverse the BDNF dysfunction in APP/PS1 mice? MiRNAs are endogenous small noncoding RNAs which play important roles in diverse biological and pathological processes, like cell differentiation, apoptosis, heart disease, neurological disorders, cancer, and so on. ${ }^{46)}$ MiR-206 is one of the known miRNAs and belongs to the miR-1 family. ${ }^{47)}$ Recently, more and more physiological effects of miR-206 are being reported. In humans there is only one mature sequence for miR-206 (has-miR-206), while in mice there are two distinct mature sequences for miR-206 (mmu-miR-206-3p, mmu-miR-206-5p). Mmu-miR-206-3p is homological to has-miR-206, and also the major form of miR-206 in mice. Although there are numerous kinds of miRNAs in the brain, in our study, the most important reason for considering miR-206 comes from previous findings which have shown that miR-206 contributes to AD by suppressing the BDNF expression in the brain. ${ }^{26-28)}$ Here, the finding that donepezil treatment decreased the hippocampal and cortical miR-206-3p expression is very interesting, extending the understanding of the pharmacological effects of donepezil, and suggests that miR-206 could be a novel pharmacological target for developing anti-dementia drugs. The miR-206-5p results are also interesting. As a minor form of miR-206 in mice, maybe the physiological role of miR-206-5p is not as wide as miR-206-3p.

AgomiRs are molecularly-modified oligonucleotides widely 

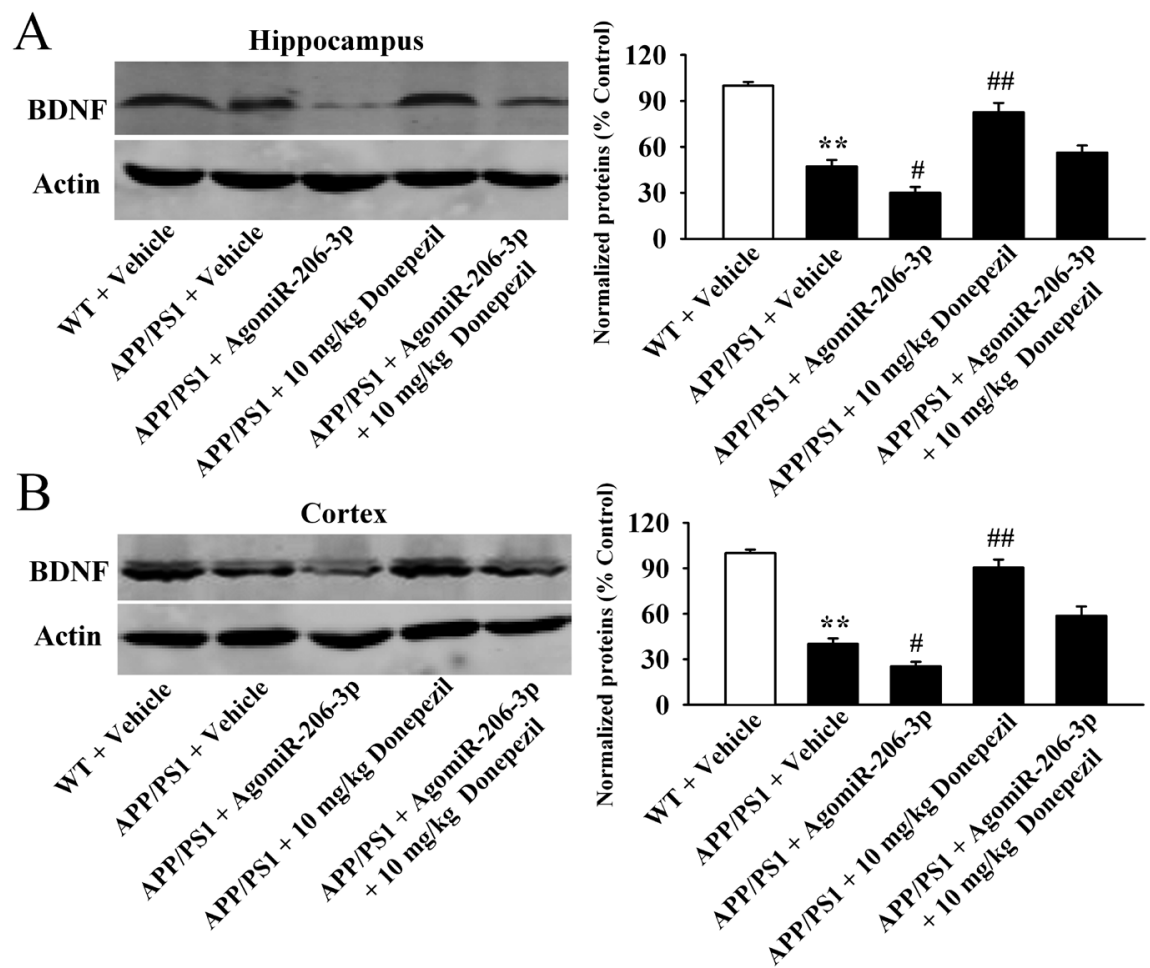

Fig. 6. The Usage of AgomiR-206-3p Attenuates the Effects of Donepezil on BDNF Protein Expression

(A) Western blotting results showed that AgomiR-206-3p pretreatment significantly prevented the donepezil-induced increase of hippocampal BDNF expression in APP/PS1 mice. (B) Western blotting results revealed that AgomiR-206-3p pretreatment also significantly prevented the donepezil-induced increase of cortical BDNF expression in APP/PS1 mice. Data are expressed as means \pm S.E.M. $(n=5) ;{ }^{*} p<0.01 v s$. WT+Vehicle; ${ }^{\#} p<0.05,{ }^{\#} p<0.01 v s$. APP/PS1+Vehicle.

used in studies of miRNAs. Here, though donepezil administration decreased the hippocampal and cortical miR-206-3p expression in APP/PS1 mice, it is not enough to conclude that the anti-dementia effects of donepezil require miR-206-3p, and so we used AgomiR-206-3p. The results that AgomiR-206-3p further exacerbated the memory impairments and BDNF dysfunction in APP/PS1 mice suggest the effectiveness of AgomiR-206-3p used in this study. Importantly and as expected, AgomiR-206-3p usage blocked the anti-dementia effects of donepezil, confirming our speculation.

In addition to $\mathrm{AD}$, miR-206 is reported to be involved in many other disorders: miR-206 is up-regulated in a rat model of myocardial infarction, and also a potential biomarker candidate for amyotrophic lateral sclerosis, and also involved in various types of cancer by regulating cell proliferation and migration, and so on. ${ }^{48-51)}$ Since donepezil treatment down-regulates the miR-206-3p expression, these reports may also extend the clinical application of donepezil, which needs more study. Moreover, in addition to miR-206-3p, the pathophysiology of AD involves many other miRNAs, like miR-106b, miR-132, miR-98, and so on. ${ }^{52-54)}$ It is possible that the anti-dementia effects of donepezil may also involve these miRNAs, and this needs further study.

Acknowledgments This work was supported by the Grant from the National Natural Science Foundation of China to Dr. Bo Jiang (No. 81401116) and Dr. Yu Chen (No. 81403127), the grants from the Provincial Natural Science Foundation of Jiangsu Province (China) to Dr. Bo Jiang (No. 14KJB310013; No. BK20161284).
Conflict of Interest The authors declare no conflict of interest.

\section{REFERENCES}

1) Price DL, Sisodia SS, Gandy SE. Amyloid beta amyloidosis in Alzheimer's disease. Curr. Opin. Neurol., 8, 268-274 (1995).

2) Hardy J, Selkoe DJ. The amyloid hypothesis of Alzheimer's disease: progress and problems on the road to therapeutics. Science, 297, $353-356$ (2002)

3) Godyń J, Jonczyk J, Panek D, Malawska B. Therapeutic strategies for Alzheimer's disease in clinical trials. Pharmacol. Rep., 68, 127-138 (2016).

4) Jia Q, Deng Y, Qing H. Potential therapeutic strategies for Alzheimer's disease targeting or beyond beta-amyloid: insights from clinical trials. Biomed. Res. Int., 2014, 837157 (2014).

5) Huang HC, Jiang ZF. Accumulated amyloid-beta peptide and hyperphosphorylated tau protein: relationship and links in Alzheimer's disease. J. Alzheimer's Dis., 16, 15-27 (2009).

6) Gouras GK, Almeida CG, Takahashi RH. Intraneuronal Abeta accumulation and origin of plaques in Alzheimer's disease. Neurobiol. Aging, 26, 1235-1244 (2005)

7) Resende R, Moreira PI, Proenca T, Deshpande A, Busciglio J, Pereira C, Oliveira CR. Brain oxidative stress in a triple-transgenic mouse model of Alzheimer's disease. Free Radic. Biol. Med., 44, 2051-2057 (2008).

8) Delay C, Hebert SS. MicroRNAs and Alzheimer's disease mouse models: current insights and future research avenues. Int. J. Alzheimer's Dis., 2011, 894938 (2011).

9) Mizuno M, Yamada K, Olariu A, Nawa H, Nabeshima T. Involvement of brain-derived neurotrophic factor in spatial memory formation and maintenance in a radial arm maze test in rats. J. Neurosci., 20, 7116-7121 (2000). 
10) Xu B, Gottschalk W, Chow A, Wilson RI, Schnell E, Zang K, Wang D, Nicoll RA, Lu B, Reichardt LF. The role of brain-derived neurotrophic factor receptors in the mature hippocampus: modulation of long-term potentiation through a presynaptic mechanism involving TrkB. J. Neurosci., 20, 6888-6897 (2000).

11) Miyata K, Omori N, Uchino H, Yamaguchi T, Isshiki A, Shibasaki F. Involvement of the brain-derived neurotrophic factor/TrkB pathway in neuroprotecive effect of cyclosporin A in forebrain ischemia. Neuroscience, 105, 571-578 (2001).

12) Yamada K, Nabeshima T. Brain-derived neurotrophic factor/TrkB signaling in memory processes. J. Pharmacol. Sci., 91, 267-270 (2003).

13) Yoshii A, Constantine-Paton M. Postsynaptic BDNF-TrkB signaling in synapse maturation, plasticity, and disease. Dev. Neurobiol., 70, 304-322 (2010).

14) Phillips HS, Hains JM, Armanini M, Laramee GR, Johnson SA, Winslow JW. BDNF mRNA is decreased in the hippocampus of individuals with Alzheimer's disease. Neuron, 7, 695-702 (1991).

15) Hock C, Heese K, Hulette C, Rosenberg C, Otten U. Region-specific neurotrophin imbalances in Alzheimer's disease: decreased levels of brain-derived neurotrophic factor and increased levels of nerve growth factor in hippocampus and cortical areas. Arch. Neurol., 57, 846-851 (2000).

16) Connor B, Young D, Yan Q, Faull RL, Synek B, Dragunow M. Brain-derived neurotrophic factor is reduced in Alzheimer's disease. Brain Res. Mol. Brain Res., 49, 71-81 (1997).

17) Zhang L, Fang Y, Lian Y, Chen Y, Wu T, Zheng Y, Zong H, Sun L, Zhang R, Wang Z, Xu Y. Brain-derived neurotrophic factor ameliorates learning deficits in a rat model of Alzheimer's disease induced by abeta1-42. PLOS ONE, 10, e0122415 (2015).

18) Mesulam MM. Neuroplasticity failure in Alzheimer's disease: bridging the gap between plaques and tangles. Neuron, 24, 521-529 (1999).

19) Lukiw WJ. Amyloid beta (Abeta) peptide modulators and other current treatment strategies for Alzheimer's disease (AD). Expert Opin. Emerg. Drugs, 17, 43-60 (2012).

20) Wilkinson DG, Francis PT, Schwam E, Payne-Parrish J. Cholinesterase inhibitors used in the treatment of Alzheimer's disease: the relationship between pharmacological effects and clinical efficacy. Drugs Aging, 21, 453-478 (2004).

21) Leyhe T, Stransky E, Eschweiler GW, Buchkremer G, Laske C. Increase of BDNF serum concentration during donepezil treatment of patients with early Alzheimer's disease. Eur. Arch. Psychiatry Clin. Neurosci., 258, 124-128 (2008).

22) Alvarez XA, Alvarez I, Iglesias O, Crespo I, Figueroa J, Aleixandre M, Linares C, Granizo E, Garcia-Fantini M, Marey J, Masliah E, Winter S, Muresanu D, Moessler H. Synergistic increase of serum BDNF in Alzheimer's patients treated with cerebrolysin and donepezil: association with cognitive improvement in ApoE4 cases. Int. J. Neuropsychopharmacol., 19, pyw024 (2016).

23) Autio H, Matlik K, Rantamaki T, Lindemann L, Hoener MC, Chao M, Arumae U, Castren E. Acetylcholinesterase inhibitors rapidly activate Trk neurotrophin receptors in the mouse hippocampus. Neuropharmacology, 61, 1291-1296 (2011).

24) Felekkis K, Touvana E, Stefanou C, Deltas C. microRNAs: a newly described class of encoded molecules that play a role in health and disease. Hippokratia, 14, 236-240 (2010).

25) McCarthy JJ. MicroRNA-206: the skeletal muscle-specific myomiR. Biochim. Biophys. Acta, 1779, 682-691 (2008).

26) Xie B, Liu Z, Jiang L, Liu W, Song M, Zhang Q, Zhang R, Cui D, Wang $\mathrm{X}, \mathrm{Xu}$ S. Increased serum miR-206 level predicts conversion from amnestic mild cognitive impairment to Alzheimer's disease: A 5-year follow-up study. J. Alzheimer's Dis., 55, 509-520 (2016).

27) Lee ST, Chu K, Jung KH, Kim JH, Huh JY, Yoon H, Park DK, Lim JY, Kim JM, Jeon D, Ryu H, Lee SK, Kim M, Roh JK. miR-206 regulates brain-derived neurotrophic factor in Alzheimer's disease model. Ann. Neurol., 72, 269-277 (2012).

28) Tian N, Cao Z, Zhang Y. MiR-206 decreases brain-derived neurotrophic factor levels in a transgenic mouse model of Alzheimer's disease. Neurosci. Bull., 30, 191-197 (2014).

29) Jiang B, Song L, Huang C, Zhang W. P7C3 Attenuates the scopolamine-induced memory impairments in $\mathrm{C} 57 \mathrm{BL} / 6 \mathrm{~J}$ mice. Neurochem. Res., 41, 1010-1019 (2016).

30) Jiang B, Huang C, Chen XF, Tong LJ, Zhang W. Tetramethylpyrazine produces antidepressant-like effects in mice through promotion of BDNF signaling pathway. Int. J. Neuropsychopharmacol., 18, pyv010 (2015).

31) Jiang B, Huang C, Zhu Q, Tong LJ, Zhang W. WY14643 produces anti-depressant-like effects in mice via the BDNF signaling pathway. Psychopharmacology, 232, 1629-1642 (2015).

32) Jiang B, Song L, Wang CN, Zhang W, Huang C, Tong LJ. Antidepressant-like effects of GM1 ganglioside involving the BDNF signaling cascade in mice. Int. J. Neuropsychopharmacol., 19, pyw046 (2016).

33) Xu H, You Z, Wu Z, Zhou L, Shen J, Gu Z. WY14643 attenuates the scopolamine-induced memory impairments in mice. Neurochem. Res., 41, 2868-2879 (2016).

34) Sun Y, Gui H, Li Q, Luo ZM, Zheng MJ, Duan JL, Liu X. MicroRNA-124 protects neurons against apoptosis in cerebral ischemic stroke. CNS Neurosci. Ther., 19, 813-819 (2013).

35) Ni YF, Zhang W, Bao XF, Wang W, Song L, Jiang B. GM1 ganglioside reverses the cognitive deficits induced by MK801 in mice. Behav. Pharmacol., 27, 451-459 (2016).

36) Yang X, Yang Q, Wang X, Luo C, Wan Y, Li J, Liu K, Zhou M, Zhang C. MicroRNA expression profile and functional analysis reveal that miR-206 is a critical novel gene for the expression of BDNF induced by ketamine. Neuromolecular Med., 16, 594-605 (2014)

37) Bao YP, Yi Y, Peng LL, Fang J, Liu KB, Li WZ, Luo HS. Roles of microRNA-206 in osteosarcoma pathogenesis and progression. Asian Pac. J. Cancer Prev., 14, 3751-3755 (2013).

38) Jiang B, Xiong Z, Yang J, Wang W, Wang Y, Hu ZL, Wang F, Chen JG. Antidepressant-like effects of ginsenoside Rg1 are due to activation of the BDNF signalling pathway and neurogenesis in the hippocampus. Br. J. Pharmacol., 166, 1872-1887 (2012).

39) Zhao L, Wang JL, Liu R, Li XX, Li JF, Zhang L. Neuroprotective, anti-amyloidogenic and neurotrophic effects of apigenin in an Alzheimer's disease mouse model. Molecules, 18, 9949-9965 (2013).

40) Hsiao YH, Hung HC, Chen SH, Gean PW. Social interaction rescues memory deficit in an animal model of Alzheimer's disease by increasing BDNF-dependent hippocampal neurogenesis. J. Neurosci., 34, 16207-16219 (2014).

41) Deardorff WJ, Grossberg GT. Pharmacotherapeutic strategies in the treatment of severe Alzheimer's disease. Expert Opin. Pharmacother., 17, 1789-1800 (2016).

42) Guo HB, Cheng YF, Wu JG, Wang CM, Wang HT, Zhang C, Qiu $\mathrm{ZK}, \mathrm{Xu}$ JP. Donepezil improves learning and memory deficits in APP/PS1 mice by inhibition of microglial activation. Neuroscience, 290, 530-542 (2015)

43) Zhang ZX, Zhao RP, Wang DS, Wang AN. Fuzhisan ameliorates Abeta production and tau phosphorylation in hippocampal of 11 month old APP/PS1 transgenic mice: A Western blot study. Exp. Gerontol., 84, 88-95 (2016).

44) Ye CY, Lei Y, Tang XC, Zhang HY. Donepezil attenuates Abetaassociated mitochondrial dysfunction and reduces mitochondrial Abeta accumulation in vivo and in vitro. Neuropharmacology, 95, 29-36 (2015).

45) Solntseva EI, Kapai NA, Popova OV, Rogozin PD, Skrebitsky VG. The involvement of sigmal receptors in donepezil-induced rescue of hippocampal LTP impaired by beta-amyloid peptide. Brain Res. Bull., 106, 56-61 (2014).

46) Li M, Marin-Muller C, Bharadwaj U, Chow KH, Yao Q, Chen C. 
MicroRNAs: control and loss of control in human physiology and disease. World J. Surg., 33, 667-684 (2009).

47) Ma G, Wang Y, Li Y, Cui L, Zhao Y, Zhao B, Li K. MiR-206, a key modulator of skeletal muscle development and disease. Int. J. Biol. Sci., 11, 345-352 (2015).

48) Shan ZX, Lin QX, Fu YH, Deng CY, Zhou ZL, Zhu JN, Liu XY, Zhang YY, Li Y, Lin SG, Yu XY. Upregulated expression of miR-1/miR-206 in a rat model of myocardial infarction. Biochem. Biophys. Res. Commun., 381, 597-601 (2009).

49) Toivonen JM, Manzano R, Olivan S, Zaragoza P, Garcia-Redondo A, Osta R. MicroRNA-206. a potential circulating biomarker candidate for amyotrophic lateral sclerosis. PLoS ONE, 9, e89065 (2014).

50) Jalali S, Ramanathan GK, Parthasarathy PT, Aljubran S, Galam L, Yunus A, Garcia S, Cox RR Jr, Lockey RF, Kolliputi N. Mir-206 regulates pulmonary artery smooth muscle cell proliferation and differentiation. PLOS ONE, 7, e46808 (2012).

51) Zhang T, Liu M, Wang C, Lin C, Sun Y, Jin D. Down-regulation of MiR-206 promotes proliferation and invasion of laryngeal cancer by regulating VEGF expression. Anticancer Res., 31, 3859-3863 (2011).

52) Liu W, Zhao J, Lu G. miR-106b inhibits tau phosphorylation at Tyr18 by targeting Fyn in a model of Alzheimer's disease. Biochem. Biophys. Res. Commun., 478, 852-857 (2016).

53) Salta E, Sierksma A, Vanden Eynden E, De Strooper B. miR-132 loss de-represses ITPKB and aggravates amyloid and TAU pathology in Alzheimer's brain. EMBO Mol. Med., 8, 1005-1018 (2016).

54) Li Q, Li X, Wang L, Zhang Y, Chen L. miR-98-5p acts as a target for Alzheimer's disease by regulating abeta production through modulating SNX6 expression. J. Mol. Neurosci., 60, 413-420 (2016). 